\title{
HEURISTICS FOR THE SOLUTION OF A VERY LARGE STREET ROUTING PROBLEM WITH MIXED TRANSPORTATION MODE
}

Servicing a large number of customers in a city zone is often a considerable part of many logistics chains. This problem is called a Street Routing Problem (SRP). As presented, only using systems such as Geographical Information Systems (GIS) it is possible to effectively manage SRP. New heuristic for solving a very large SRP is evaluated on the real data. One of the key properties of GIS for use with the routing software is its flexible interactive and user-friendly environment. The paper presents several approximations of length for SRP with mixed transportation mode.

Keywords: SRP, VRP, GIS, heuristics, approximations

\section{Introduction}

The Street Routing Problem (SRP), as a problem of servicing a large number of customers in a city zone or several connected cities is often a part of many logistics chains. In this paper we will mostly address a special case of SRP that is a postal service delivery. A typical SRP uses samples with thousands of customers. We want to evaluate and find solutions for cases with more than 10000 customers. In such agglomerations service is done using a mixed transportation mode. The service personnel is driven by a car or uses public transportation to a first point of the service area, then serves the area usually on foot and then returns to the depot using either public transportation or a car. In this paper we will focus on the transport of personnel by cars. To find a good solution for these cases we must be able to approximate the length of SRP. We need to aggregate customers to natural clusters and solve a special case of the Capacitated Vehicle Routing Problem (CVRP) for finding good routes of cars.

\section{Experimental sample}

The SRP is a less explored group of problems than the Vehicle Routing Problem (VRP) and there is not a good, publicly available sample of data for the method comparison. Authors, for example [7], are usually solving specific problems and it is difficult to compare solutions across these problems because each of them is somewhat unique.

It is necessary to use our own experimental sample, one that covers most of the typical SRP and, at the same time, it is possible to compare each of the solution methods. Our experimental sample was from four large cities and surrounded villages in Slovakia.
Customers were houses in these cities and full street infrastructure is available. These cities were Bratislava, Kosice, Zilina, Presov. A number of customers for these four agglomerations vary from 12000 to 29000 .

The data were collected manually from the source maps ZM 1:10 000, purchased from the Geodetic and Cartographic Institute of the Slovak Republic. Some data were collected using GPS receivers. These data are not publicly available at the moment.

\section{Definition of a very large SRP with mixed transportation mode}

There are cases of serving a large number of customers from one central depot. Centralizing all delivery operations to one depot for the large number of customers has some advantages and disadvantages. One of the key advantages to centralization is the ability to use more advanced and expensive technologies for preparation before the deliveries are picked up by the postmen. The preparation of deliveries usually takes more than $10 \%$ of the total working time of postmen. By shortening this time combined with good transportation methods for placing the postmen in their districts we allow more time for a delivery.

Centralizing delivery operations creates a large aggregation of customers that need to be served from one depot. In Fig. 1 we present a possible area of the served region around the city of Zilina with 12663 customers.

Our goal is to find a good solution for the SRP in such a large, centralized region. For each customer we have an average service time. Postmen can be driven by car to the starting point of the

\footnotetext{
* Peter Matis

Department of Transport Systems, Faculty of Managements and Informatics, University of Zilina, Slovakia, E-mail: Peter.Matis@uniza.sk
} 


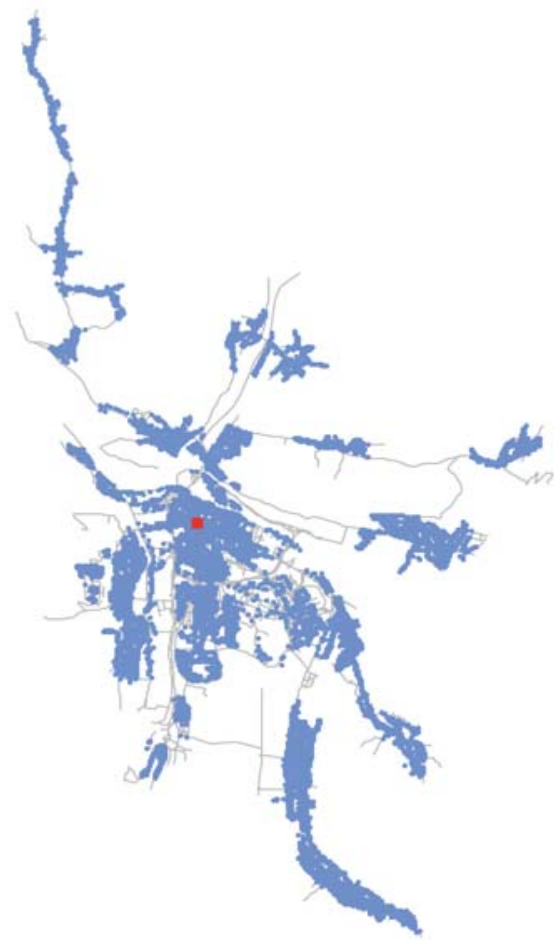

Fig. 1: Customers and depot in large Zilina region

service and at the end of the working day they can be driven back to the depot. Deliveries are made on foot, so the problem represents a special case of SRP with a mixed transportation mode. The car driver is also a postman and serves one district. There are no transportation related expenses. The delivery time is limited for each postman. Time gaps between the physical end of deliveries and the car arrival to drive the postman back should be tide.

\section{The aggregation of customers to natural clusters and approximation of a route length}

One of the key problems in solving a very large SRP with a mixed transportation mode is to aggregate customers to natural clusters. Each cluster is then treated as one district for the SRP and is served by one postman. The size of the cluster is determined by an estimated length of the SRP route, the travel speed of the postman, the delivery time for customers in the cluster and the total time available for serving the cluster.

There is one problem related to the fact that one logic city unit (vicinity village or city district) can be divided to several service districts and the last service district can take only a small amount of the postman's working time. It is hard to merge these residuum districts to one district, because they can be distributed across the whole studied area.

For aggregation of customers to natural clusters we used a part of Fuzzy Cluster Heuristics (FCH) [5]. This heuristics can be implemented in the following steps:
1. Estimate the minimum number of clusters $p$ needed for serving all the customers using the following formula

$p=\downarrow\left(\frac{\sum_{c=1}^{N} C S T_{c}+\frac{\sum_{s=1}^{M} D_{s}}{V}}{S W T}\right)$

where symbol $\downarrow$ represents nearest smaller integer number, $C S T_{c}$ is a service time for the customer $c, N$ is a number of customers, $M$ is a number of street segments where customers are located, $D_{s}$ is a length of street segment $s, V$ is an average speed of the postman walking on foot, $S W T$ is the available working time of one postman for service of customers.

2. Locate $p$ medians, so they are uniformly distributed in the serviced area.

3. Create $p$ clusters of customers around these medians using clustering by "fuzzy c-means" (FCM). The membership of customers in each cluster is set as a triangle fuzzy number. The triangle fuzzy number is based on route distance between the cluster median and customer.

4. For each cluster approximate the route length and estimate the average service time.

5. If there are many clusters (more than $20 \%$ ) which have their service time over the time SWT, increase the number of clusters $p$ and go to step 2, otherwise end the algorithm with the resulting clusters and these are used as SRP districts.

In this algorithm, we used an approximation of length for the SRP route of one postman. In our case this is actually the length of TSP or special TSP, where the postman does not necessarily need to return back to the point where his route started.

In literature there are some good samples as to how other authors estimate the length of TSP or VRP [1]. We present here two estimations from Toth, Vigo and Kwon et al.

$$
\begin{aligned}
\operatorname{TSP}(D) & =0.765 \sqrt{N A} \\
\operatorname{TSP}(D) & =[0.83-0.0011(N+1)+ \\
& +1.11 S /(N+1)] \sqrt{N A}
\end{aligned}
$$

where $\operatorname{TSP}(D)$ is the estimated length of the route, $A$ is an area of a serviced cluster, $S$ is a proportion of the length-width ratio for the outside rectangle of the cluster created such a way that the ratio is always larger than or equal to 1 . The authors always use the Euclidean or Manhattan travel metric.

In our case neither Euclidian nor Manhattan metrics interpret the real distance for the traveling salesman. Our case is specific because the authors focus on the VRP and our problem is the SRP. The SRP has a couple of differences to VRP [3] and some of these differences are related to a distribution of customers and the network density. In our model we used the following estimation of the length for one postman's path:

$\operatorname{TSP}(D)=1.31 * S D$ 
where $S D$ is a total distance of all the street segments that are served by one postman. This formula was created from a simple regression using sample data with 125 service clusters compared to the best results from heuristics described by Matis [5]. Figure 2 represents use FCH in one large SRP case.

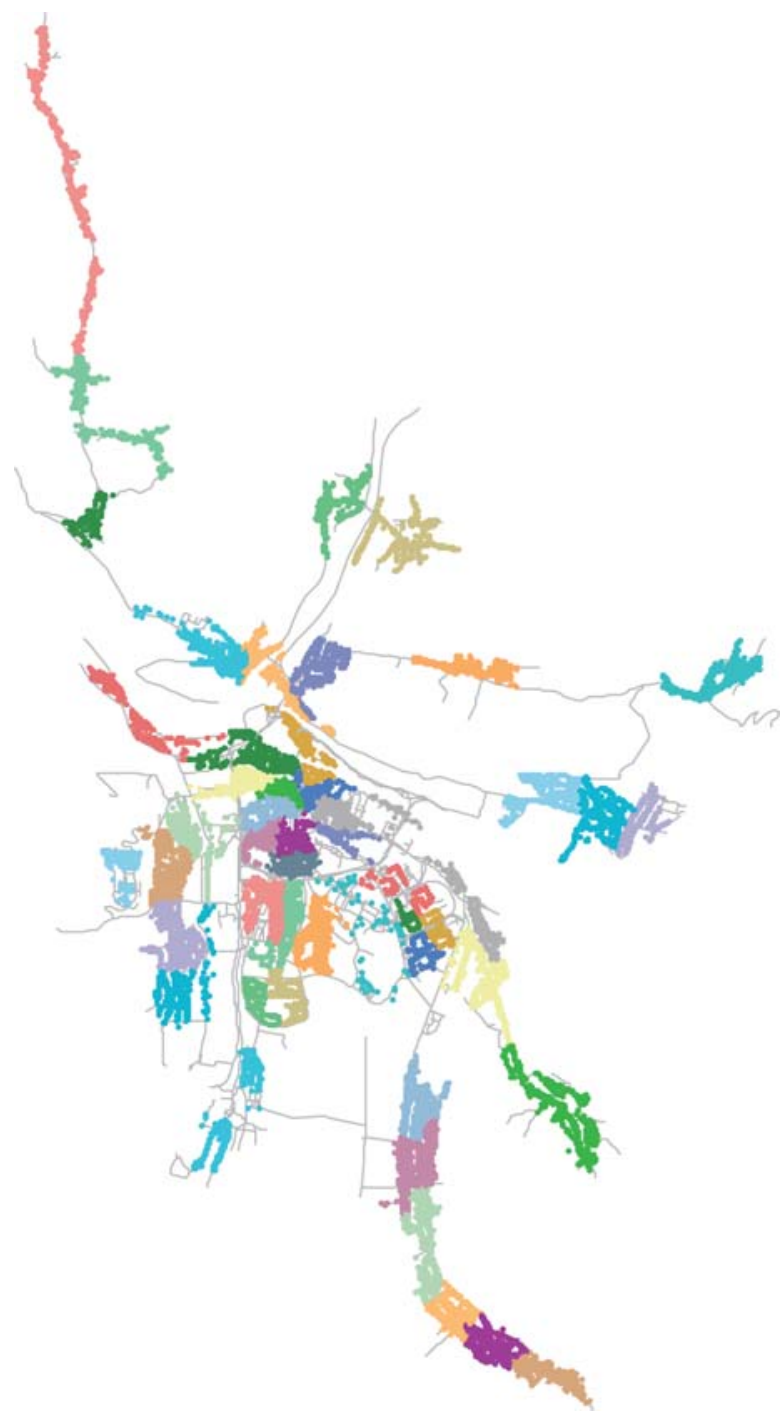

Fig. 2: Natural clusters in large Zilina region created by FCH

\section{Transportation of postmen to districts}

To get a solution for a very large SRP we need to distribute postmen to their service districts using cars. This is a variation of a normal Capacitated Vehicle Routing Problem (CVRP) with addition of an extra condition that we have to maximize utilization of vehicles. An additional condition is that the vehicle has to return to the depot using the same route. In our case all the vehicles have the same capacity of 5 postmen. The goal is to minimize the total length of routes for all the cars and, second, to minimize a number of cars that are necessary for all the service districts that are farther from the service center - the post office building. The car's driver serves the last district and then collects all the postmen that were in his car when they started in the service center. The postman that first leaves the car has more time for delivery than the last postman in the car. Knowing this we could change our algorithm for a creation of natural clusters, and make clusters that are closer to the service center larger and further clusters made smaller. In our case, we did not make this change. The difference in time when they leave the car is usually less than 10 minutes and because the delivery time has a probabilistic character, this may not have affect on the final result.

There is no method that could find an optimal solution for this problem for a practical size SRP. We used Unified Cluster First Route Second (UCFRS) heuristics [5] that was changed to this specific case where our goal was not only to find the minimum total length, but also to maximize the utilization of the car. Heuristics was named as Unified Cluster First Route Second for Postman Collection heuristics (UCFRSPC).

A major difference between the original UCFRS and the UCFRSPC is that a number of serviced clusters in the route is limited by a maximum number of passengers of the car. For the one trip's length calculation we use only lengths of segments from the depot to the last customer. The car has to return back to the depot using the same route. The UCFRSPC heuristics executes the following steps.

1. Mark all the centers of clusters (CC) as not visited.

2. Pick from all not visited $\mathrm{CC}$ the furthest one. Set the route's center equal to the position of the first picked CC.

3. If the number of $\mathrm{CC}$ in the current route is equal to the capacity of a car go to step 5 .

4. Choose the closest CC to the route's center (using road distances). Put this CC to the current route and recalculate the position of the route's center as the closest point on the transportation network to the gravity point of all the CCs in the current route. Mark the $\mathrm{CC}$ as visited. Go to step 3.

5. If there are some not visited $\mathrm{CC}$ then change the route number to a new number and go to step 2, otherwise calculate the minimum total length for every route. The sum of these lengths is the total length of all the car trips.

Fig. 3 shows the resulting routes for $40 \mathrm{CC}$ divided to 8 cars.

We calculate the minimum total length for every route evaluation of all permutation of possible orders of $\mathrm{CC}$ in the route. We can do it, because the size of the CC set is a small, usually there are $5 \mathrm{CC}$ in one route.

\section{Use of GIS as decision support system for the SRP}

Recent development in the real street routing problems shows that there is a need to make routing software a part of a larger system. One of possible solutions to this is to integrate routing 


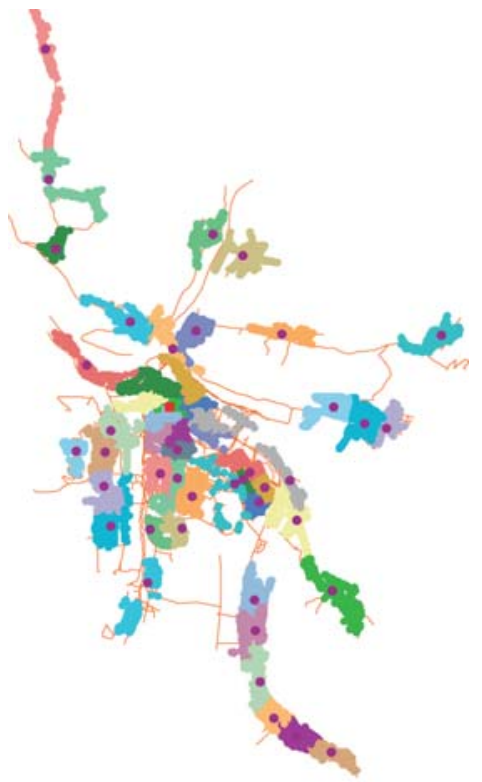

software within GIS. GIS can be helpful in the collection, storing and management of large geographical databases used in the routing software. GIS can also be used for the creation of all outputs from the routing software. One of the key properties of GIS for the use of routing software is the interactive and user-friendly environment. Routing software can find a good solution and explore possibilities and an expert can change calculated routes to explore other possibilities based on the expert's judgment.

GIS has several useful features that could help improve the routing software performance. To mention only a few here - we may pay attention to the capabilities as safe database management, flexible symbols, map management, drawing capabilities, safety and interoperability.

Decisions support systems (DSS) are important for solving real SRPs. Software can be used for a solution of the problem, and also for exploring several other possibilities, or seeing how changes in the transportation network, regulations or policies could affect the routes, expenses and other parameters. Currently there exist two trends in the development of DSS for SRP.

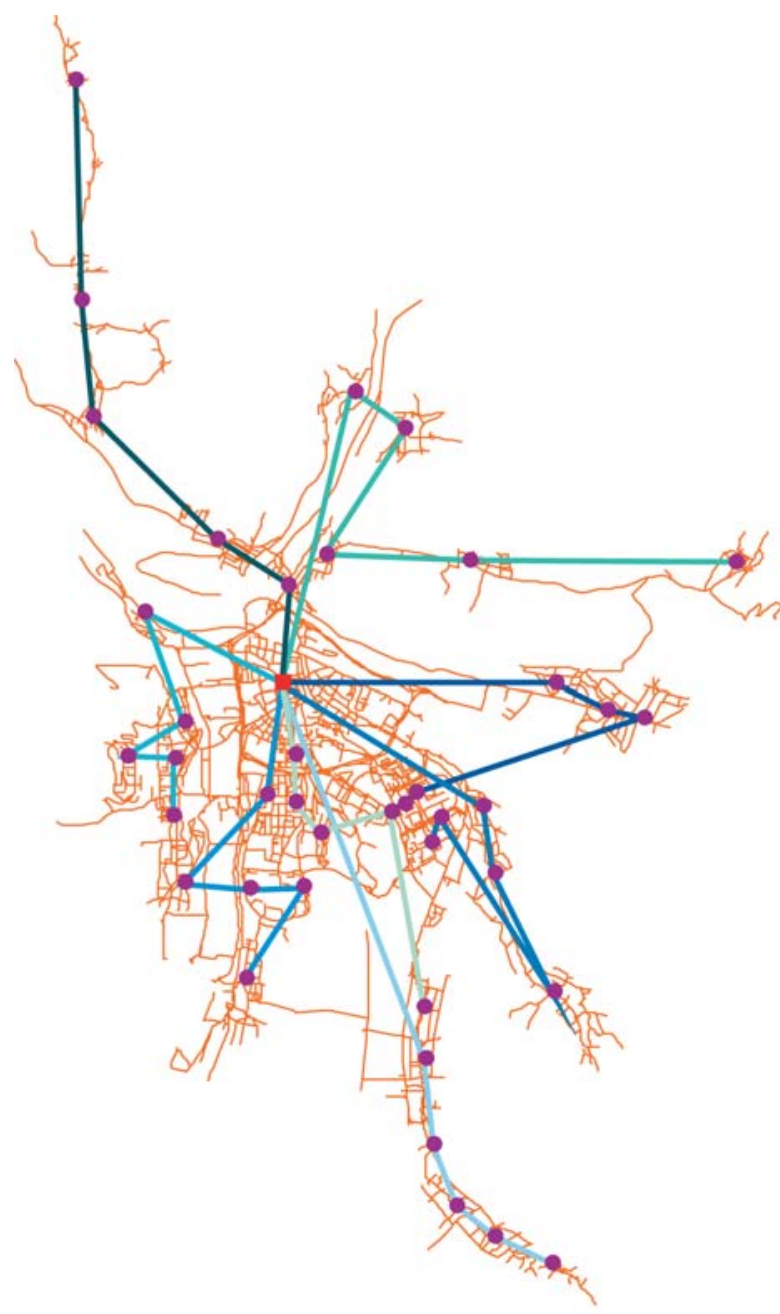

- Independent software packages specialized for SRP with limited amount of DSS capabilities. Here we can note software like GeoRoute [8] from the Canadian software firm GIRO. It is not an open system. It is an expensive product that costs 1,000,000 EUR for one installation. As an example of other such systems, we can use TRANSCAD [9] created in the US, which has some similarities to GeoRoute. GeoRoute is more feasible for solving the SRP because it is specialized for the street routing. TRANSCAD is more specialized in the node routing.

- Integrated systems based on the GIS or CAD. As an example, we can cite ArcView [10] with its ArcGIS Network Analyst. It specializes in the node routing. The user can implement extension and then make it an integral part of the whole system.

An important feature of a decision support system is good visualization and a good editor. Tools that are able to visualize results are easily acceptable to users. Visualization also allows users to see any problems or discrepancies which are not easy to find or implement by heuristics. The user can change starting conditions in the problem to avoid these discrepancies, test the new model, and by several iterations, get an acceptable result.

To shorten development time, we have chosen a standard GIS environment as the base system for the data management. The integrator represents the main user interface. It controls each heuristic - the connection of GIS database to/from heuristics. In some cases the heuristics could be replaced by regular solvers, XPRESS for example. We used ArcView by ESRI as the main GIS tool, because it is an open system and it allows the user to program its functionalities. The GIS is managed from the integrator by the Avenue scripting language, $\mathrm{C \#}$, and VBasic, depending on the version of ArcView. The GIS is used in the system as a data management tool, editor, for visualization of results.

Fig. 3: Centers for natural clusters in the large Zilina region served by cars and optimal routes for 8 cars 


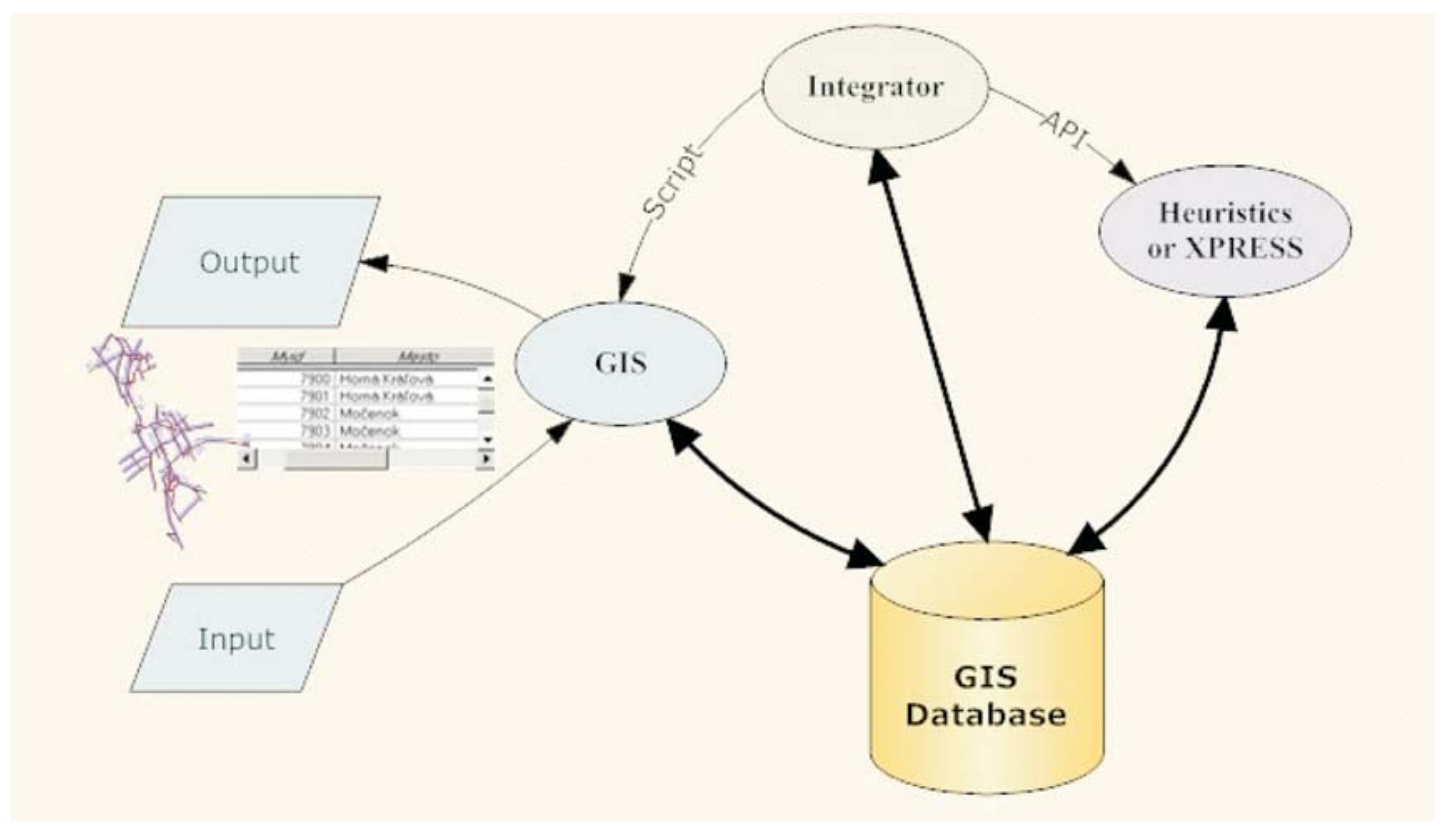

Fig. 4: Decision Support System using of GIS for SRP

\section{Conclusions}

There are expanding activities in the cities that can be presented as street-based tasks. We introduced some methodology for solving a specific SRP with a very large volume of customers. We used the samples from four cities for verification of our methodology and algorithms. This problem is not documented in the literature.

We presented a new heuristic for creation of natural clusters and estimation of the route's length. We have not compared the results of the heuristics with any other results because there is no literature dealing with this problem. From the small sample we can conclude that an average calculation time for this part of the solu- tion is 232sec using a PC with Dual Core Processor Intel Pentium. For most of the practical applications this is acceptable and results are promising.

We created a new heuristics for the distribution of postmen to their service districts using the cars. The results show that heuristics can be used also for the cases with a heterogeneous vehicle park. This is very fast heuristics.

We created DSS for solving these specific SRP which was tested only on the sample data.

Acknowledgement: This work has been supported by the grant VEGA 1/0591/09.

\section{References}

[1] FIGLIOZZI, M. A.: Planning Approximations to the Average Length of Vehicle Routing Problems with Varying Customer demands and Routing Constraints. Transportation Research Record: Journal of the Transportation Board, No 2089, p. 1-8.

[2] JANACEK, J.: Optimization in transport networks (in Czech), EDIS-ZU, Zilinska univerzita, Zilina, 2003, 248 p.

[3] MATIS, P.: Management of street routing problems using decisions support system, Communications 3, Zilinska univerzita, Zilina, 2006, p. 5-8

[4] MATIS, P.: The relationship between quantitative and qualitative measurements in solving of street routing problems, $15^{\text {th }}$ Internatinal Scientific Conference on Mathematical Methods in Economics and Industry, Herlany, 2007, p. 144-152.

[5] MATIS, P.: Decision support system for solving the street routing problem, TRANSPORT 2008 23(3), ISSN 1648-4142, p. 230-236.

[6] POOT, A., KANT, G., WAGELMANS, A.: A Saving based method for real-life vehicle routing problems, Journal of the Operational Research Society, Vol. 53, 2002, p. 57-68

[7] RUIZ, R., MAROTO, C., ALCARAZ, J.: A decision support system for a real vehicle routing problem, European Journal of Operational Research 153, 2004, p. 593-606.

[8] GeoRoute, http://www.giro.ca/en/products/georoute/index.htm

[9] TransCAD, http://www.caliper.com/tcovu.htm

[10] ArcView, http://www.esri.com/software/arcview/ 